\title{
ON MATRIX SUPERPOTENTIAL AND THREE-COMPONENT NORMAL MODES
}

\author{
R. de Lima Rodrigues ${ }^{(a)}$, A. F. de $\operatorname{Lima}^{(b)}$, \\ E. R. Bezerra de Mello ${ }^{(c)}$, and V. B. Bezerra ${ }^{(c)}$ \\ ${ }^{(a)}$ Centro Brasileiro de Pesquisas Físicas \\ 22290-180, Rio de Janeiro-RJ, Brazil \\ (b) Departamento de Física, Universidade Federal de Campina Grande \\ 58109-790 Campina Grande, PB, Brazil \\ (c) Departamento de Física, Universidade Federal da Paraíba \\ 58051-970, João Pessoa, PB, Brazil
}

\begin{abstract}
We consider the supersymmetric quantum mechanics(SUSY QM) with three-component normal modes for the Bogomol'nyi-Prasad-Sommerfield (BPS) states. An explicit form of the SUSY QM matrix superpotential is presented and the corresponding three-component bosonic zero-mode eigenfunction is investigated.
\end{abstract}

Keywords: Superpotential, BPS states, stability equation.

PACS numbers: 11.30.Pb, 03.65.Fd, 11.10.Ef.

Permanent address of RLR: Unidade Acadêmica de Educação, Universidade Federal de Campina Grande, Cuité - PB, 58.175-000, Brazil.

E-mails: $\quad$ rafael@df.ufcg.edu.br, aerlima@df.ufcg.edu.br, emello@fisica.ufpb.br, valdir@fisica.ufpb.br 


\section{INTRODUCTION}

Supersymmetric quantum mechanics(SUSY QM) has provided a possibility to solve analytically some non-relativistic quantum systems. The simplest model in this framework was proposed by Witten[1] in the early eights of the last Century. After this pioneered work, the methods of SUSY QM have quickly developed and some applications arised. Some of these applications include the problems related with the superpotential [2, 3], whose generalization to the case of a matrix superpotential was done a long time ago. The SUSY QM has also applications in the non-relativistic quantum context [4]-[7] and in the case involving two or three fields in $(1+1)$-dimensional model. Others applications include some results connected with self-adjoint extensions [8] and superpotential matrix [9], among others.

The classical configurations with domain wall solutions are bidimensional structures in $(3+1)$-dimensions [10]-[14]. They are static, non-singular, classically stable Bogomol'nyi [15] and Prasad-Sommerfield [16] (BPS) soliton (defect) configurations, with finite localized energy associated with a real scalar field potential model.

The BPS states are classical configurations that satisfy first and second order differential equations. In a context that stresses the connection with BPS-bound states[17, 18], domain walls have been exploited.

Recently, the stability and metamorphosis of BPS states have been investigated [19], using the framework of SUSY QM, with a detailed analysis of a 2-dimensional $N=$ 2-Wess-Zumino model in terms of two chiral superfields and composite dyons in $N=2$ -

supersymmetric gauge theories[20]. Also, the superpotential associated with the linear classical stability of the static solutions for systems with one real scalar field in (1+1)-dimensions were discussed in the literature [2, 3]. However, for solitons associated with three coupled scalar fields there are no general rules for finding analytic solutions since the nonlinearity in the potential leads to an increasing of the difficulties to solve the BPS and field equations.

This paper is organized as follows: In Section II, we discuss SUSY algebra with topological charge. In Section III, we consider a SUSY model for two coupled scalar fields. In Section IV, we present the BPS configurations for three coupled scalar fields. In Section V, we define the BPS mass bound of the energy and discuss the stability of BPS states. The Schrödinger-like equation and also the Hessian matrix are obtained. In Section VI, a matrix superpotential with three-component wave functions is obtained. In Section VII, a specific 
potential model is investigated. Our conclusions are presented in Section VIII.

\section{SUPERSYMMETRY ALGEBRA WITH TOPOLOGICAL CHARGE}

Let us start with a discussion concerning central charges due to the fact that these quantities characterize SUSY. To do this, consider the potential model with one single real scalar field $\phi$, whose Lagragian is given by

$$
A=\int d^{2} z \frac{1}{2}\left\{\left(\partial_{\mu} \phi\right)^{2}+\left[\bar{\psi} \partial_{\mu} \gamma^{\mu} \psi-U^{2}(\phi)-U^{\prime}(\phi) \bar{\psi} \psi\right]\right\}
$$

where $U(\phi)$ a well defined continuous function and the Majorana spinor, $\psi(z)$, is given by

$$
\psi(z)=\left(\begin{array}{l}
\psi_{+}(z) \\
\psi_{-}(z)
\end{array}\right) .
$$

In this case, the conserved SUSY current can be written as

$$
S_{\beta}^{\mu}=\left(\partial_{\alpha} \phi\right)\left(\gamma^{\alpha} \gamma^{\mu}\right)_{\beta \xi} \psi_{\xi}+U(\phi) \gamma_{\beta \xi}^{\mu} \psi_{\xi}
$$

Therefore, the topological SUSY charge is given by

$$
Q_{\beta}=\int S_{\beta}^{0} d z
$$

and, then, we can write

$$
\begin{aligned}
& Q_{+}=\int d z\left[\left(\partial_{0} \phi+\partial_{1} \phi\right) \psi_{+}-U(\phi) \psi_{-}\right], \\
& Q_{-}=\int d z\left[\left(\partial_{0} \phi-\partial_{1} \phi\right) \psi_{-}+U(\phi) \psi_{+}\right] .
\end{aligned}
$$

In (1+1)-dimensions the SUSY algebra becomes

$$
Q_{+}^{2}=P_{+}=P_{0}+P_{1}, \quad Q_{-}^{2}=P_{-}=P_{0}-P_{1}
$$

and

$$
Q_{+} Q_{-}+Q_{-} Q_{+}=0
$$


where

$$
\begin{aligned}
{\left[\psi_{-}(y), \psi_{-}(x)\right]_{+}=} & \delta(y-x), \quad\left[\psi_{+}(y), \psi_{+}(x)\right]_{+}=\delta(y-x), \\
& {\left[\psi_{-}(x), \psi_{+}(x)\right]_{+}=0 . }
\end{aligned}
$$

In a field theory without soliton solutions these equations are satisfied. However, in a field theory with soliton solutions these equations are not satisfied because the surface terms for a soliton solution are different from zero, and as a consequence

$$
Q_{+} Q_{-}+Q_{-} Q_{+}=\int_{-\infty}^{+\infty} d z \frac{\partial}{\partial x}(2 \Gamma(\phi))
$$

with the superpotential satisfying the relation

$$
\Gamma^{\prime}(\phi)=\frac{d}{d \phi} \Gamma=U(\phi)
$$

Note that the right hand side of Eq. (9) is a scalar, which corresponds exactly to the central charge. Thus, the Bogomol'nyi classical bound, for a single particle with mass $m_{0}$, at rest, which means that, $P_{+}=P_{-}=m_{0}$, becomes

$$
m_{0} \geq\left|\int_{-\infty}^{+\infty} d z \frac{\partial}{\partial z} \Gamma(\phi)\right|=\left|\Gamma\left[M_{j}\right]-\Gamma\left[M_{i}\right]\right|,
$$

where $M_{i}$ and $M_{j}$ represent the vacuum states. It is worth calling attention to the fact that this inequality remains valid for soliton and antisoliton solutions at one-loop order.

\section{SUSY FROM TWO COUPLED SCALAR FIELDS}

Let us write the potential $V\left(\phi_{j}\right)$ in the following SUSY form, analogous to the case with one single field only,

$$
V\left(\phi_{j}\right)=\frac{1}{2}\left(U_{1}^{2}\left(\phi_{j}\right)+U_{1}^{2}\left(\phi_{j}\right)\right), \quad U_{i}\left(\phi_{j}\right)=U_{i}\left(\phi_{1}, \phi_{2}\right)
$$

Thus, the $N=1$ algebra can be discussed by investigating the SUSY Lagrangian density in $(1+1)$-dimensions with the following form

$$
\mathcal{L}=\frac{1}{2}\left(\partial_{\mu} \phi_{1}\right)^{2}+\frac{1}{2}\left(\partial_{\mu} \phi_{2}\right)^{2}+\frac{1}{2} \bar{\psi}_{1}\left(i \gamma^{\mu} \partial_{\mu}-\frac{\partial U_{1}}{\partial \phi_{1}}\right) \psi_{1}
$$




$$
\begin{aligned}
& -\frac{1}{2} U_{1}^{2}\left(\phi_{j}\right)-\frac{1}{2} U_{2}^{2}\left(\phi_{j}\right)+\frac{1}{2} \bar{\psi}_{2}\left(i \gamma^{\mu} \partial_{\mu}-\frac{\partial U_{2}}{\partial \phi_{2}}\right) \psi_{2} \\
& -\frac{1}{2} \frac{\partial U_{1}}{\partial \phi_{2}} \bar{\psi}_{1} \psi_{2}-\frac{1}{2} \frac{\partial U_{2}}{\partial \phi_{1}} \bar{\psi}_{2} \psi_{1}
\end{aligned}
$$

where $\psi_{1}$ and $\psi_{2}$ are Majorama spinors. In this framework, the SUSY current is given by

$$
S^{\mu}=\left(\partial_{\alpha} \phi_{1}\right) \gamma^{\alpha} \gamma^{\mu} \psi_{1}+i U_{1}\left(\phi_{j}\right) \gamma_{\mu} \psi_{1}+\left(\partial_{\beta} \phi_{2}\right) \gamma^{\beta} \gamma^{\mu} \psi_{2}+i U_{2}\left(\phi_{j}\right) \gamma^{\mu} \psi_{2}
$$

and therefore, the conserved supercharges can be expressed as

$$
\begin{aligned}
Q_{ \pm}= & \frac{1}{\sqrt{2}} \int d z\left\{\left(\partial_{0} \phi_{1} \pm \partial_{1} \phi_{1}\right) \psi_{ \pm} \mp U_{1}\left(\phi_{J}\right) \psi_{\mp}\right\} \\
& +\frac{1}{\sqrt{2}} \int d z\left\{\left(\partial_{0} \phi_{2} \pm \partial_{1} \phi_{2}\right) \psi_{ \pm} \mp U_{2}\left(\phi_{j}\right) \psi_{\mp}\right\} .
\end{aligned}
$$

On the other hand, the superpotential $W\left(\phi_{j}\right)$ satisfy

$$
\frac{\partial W}{\partial \phi_{1}}=U_{1}\left(\phi_{j}\right), \quad \frac{\partial W}{\partial \phi_{2}}=U_{2}\left(\phi_{j}\right)
$$

which leads to the value for a Bogomol'nyi minimum energy.

\section{CONFIGURATIOS WITH THREE COUPLED SCALAR FIELDS}

In this section, we consider classical soliton solutions with three coupled real scalar fields, $\phi_{j},(j=1,2,3)$, in $(1+1)$-dimensions included in bosonic sector and explain the equality of topological and central charges, $\psi_{i}=0$. The soliton solutions are static, nonsingular, classically stable and finite localized energy solutions of the field equations. The Lagrangian density for such nonlinear system in the natural system of units $(c=\hbar=1)$, in a $(1+1)$ dimensional space-time, with Lorentz invariance, is written as

$$
\mathcal{L}\left(\phi_{j}, \partial_{\mu} \phi_{j}\right)=\frac{1}{2} \sum_{j=1}^{3}\left(\partial_{\mu} \phi_{j}\right)^{2}-V\left(\phi_{j}\right),
$$

where $\partial_{\mu}=\frac{\partial}{\partial z^{\mu}}, \quad z^{\mu}=(t, z)$ with $\mu=0,1, \quad \phi_{j}=\phi_{j}(t, z)$ and $\eta^{\mu \nu}=\operatorname{diag}(+,-)$ is the metric tensor. Here, the potential $V\left(\phi_{j}\right)=V\left(\phi_{1}, \phi_{2}, \phi_{3}\right)$ is a positive definite function of $\phi_{j}$. The general classical configurations obey the following equation 


$$
\frac{\partial^{2}}{\partial t^{2}} \phi_{j}-\frac{\partial^{2}}{\partial z^{2}} \phi_{j}+\frac{\partial}{\partial \phi_{j}} V=0
$$

which, for static soliton solutions, is equivalent to the following system of nonlinear second order differential equations

$$
\phi_{j}^{\prime \prime}=\frac{\partial}{\partial \phi_{j}} V, \quad(j=1,2,3),
$$

where prime denotes differentiation with respect to the space variable.

There is in literature, a trial orbit method for finding static solutions of Eq.(19), for certain positive potentials. This constitutes what is termed the "trial and error" technique [11]. This method has many limitations, notably the need to choose trial orbits. Solutions had to be obtained by ingenuity combination with trial and error rather than by systematic derivation from the field equations. In this paper we will use the trial orbit method for the first order differential equations associated with three real scalar fields, differently from what was done by Rajaraman[11], who applied this method to the equation of motion.

Let us assume that the trial orbit is given by

$$
G\left(\phi_{1}, \phi_{2}, \phi_{3}\right)=0
$$

Thus, we have

$$
\frac{d}{d z} G\left(\phi_{1}, \phi_{2}, \phi_{3}\right)=\sum_{i=1}^{3} \frac{\partial G}{\partial \phi_{i}} \phi_{i}^{\prime}=0 .
$$

Taking Eqs. (20) and (21) into account, we can get the constant coefficients in such trial orbit by substitution of the vacuum and the BPS states into these equations.

\section{LINEAR STABILITY}

Since the potential $V\left(\phi_{j}\right)$ is positive, it can be written in the square form analogous to the case in which we have just one single field [2], as

$$
V\left(\phi_{j}\right)=V\left(\phi_{1}, \phi_{2}, \phi_{3}\right)=\frac{1}{2} \sum_{j=1}^{3} U_{j}^{2}\left(\phi_{1}, \phi_{2}, \phi_{3}\right), \quad U_{j}\left(\phi_{1}, \phi_{2}, \phi_{3}\right) \equiv \frac{\partial W}{\partial \phi_{j}},
$$

where $W$ is the superpotential associated with the three fields. 
Therefore, we can write the total energy given by

$$
E=\int_{-\infty}^{+\infty} d z \frac{1}{2}\left[\left(\phi_{1}^{\prime}\right)^{2}+\left(\phi_{2}^{\prime}\right)^{2}+\left(\phi_{3}^{\prime}\right)^{2}+2 V(\phi, \chi)\right]
$$

in the BPS form, which consists of a sum of squares and surface terms, as

$$
E=\int_{-\infty}^{+\infty} d z\left(\frac{1}{2}\left(\phi_{1}^{\prime}-U_{1}\right)^{2}+\frac{1}{2}\left(\phi_{2}^{\prime}-U_{2}\right)^{2}+\frac{1}{2}\left(\phi_{3}^{\prime}-U_{3}\right)^{2}+\frac{\partial}{\partial z} W\right)
$$

Note that the first three terms are always positive and thus, the lower bound of the energy is given by the fourth term, which means that

$$
E \geq\left|\int_{-\infty}^{+\infty} d z \frac{\partial}{\partial z} W\left[\phi_{1}(z), \phi_{2}(z), \phi_{3}(z)\right]\right|,
$$

where the superpotential $W=W\left[\phi_{1}(z), \phi_{2}(z), \phi_{3}(z)\right]$ will be discussed in what follows. The BPS mass bound of the energy which results in a topological charge is given by

$$
E_{B P S}=T_{i j}=\left|W\left[M_{j}\right]-W\left[M_{i}\right]\right|,
$$

where $M_{i}$ and $M_{j}$ represent the BPS vacuum states and are the extrema of $W$. In this case the BPS states satisfy the following set of first order differential equations

$$
\phi_{j}^{\prime}=U_{j}\left(\phi_{1}, \phi_{2}, \phi_{3}\right)
$$

Now, let us analyze the classical stability of the soliton solutions in this nonlinear system, taking into consideration small perturbations around $\phi_{j}(z)(j=1,2,3)$, namely, $\eta_{j}$. Thus, we can write the classical solution of the system as

$$
\phi_{j}(t, z)=\phi_{j}(z)+\eta_{j}(t, z), \quad(j=1,2,3) .
$$

We can expand the fluctuations $\eta_{j}(t, z)$ in terms of the normal modes, in the following way

$$
\eta_{j}(t, z)=\sum_{n} \epsilon_{j, n} \eta_{j, n}(z) e^{i \omega_{j, n} t}, \quad \omega_{1, n}=\omega_{2, n}=\omega_{3, n}=\omega_{n},
$$

where $\epsilon_{j, n}$ are real constant coefficients. Thus, the stability equation for the fields turns into a Schrödinger-like equation for a three-component eigenfunction $\Psi_{n}$, 


$$
\mathcal{H} \Psi_{n}=\omega_{n}^{2} \Psi_{n}, \quad n=0,1,2, \cdots
$$

where

$$
\mathcal{H}=\left(\begin{array}{ccc}
-\frac{d^{2}}{d z^{2}}+\frac{\partial^{2}}{\partial \phi_{1}^{2}} V & \frac{\partial^{2}}{\partial \phi_{1} \partial \phi_{2}} V & \frac{\partial^{2}}{\partial \phi_{1} \partial \phi_{3}} V \\
\frac{\partial^{2}}{\partial \phi_{2} \partial \phi_{1}} V & -\frac{d^{2}}{d z^{2}}+\frac{\partial^{2}}{\partial \phi_{2}^{2}} V & +\frac{\partial^{2}}{\partial \phi_{2} \partial \phi_{3}} V \\
\frac{\partial^{2}}{\partial \phi_{3} \partial \phi_{1}} V & \frac{\partial^{2}}{\partial \phi_{3} \partial \phi_{2}} V & -\frac{d^{2}}{d z^{2}}+\frac{\partial^{2}}{\partial \phi_{3}^{2}} V
\end{array}\right)_{\mid \phi_{j}=\phi_{j}(z)} \equiv-\mathbf{I} \frac{d^{2}}{d z^{2}}+V_{F}(z)
$$

with $\mathbf{I}$ being the $(3 \times 3)$-dentity matrix and $V_{F}(z)$ the $(3 \times 3)$ fluctuation Hessian matrix. The excited modes are, thus, given by

$$
\Psi_{n}(z)=\left(\begin{array}{c}
\eta_{1, n}(z) \\
\eta_{2, n}(z) \\
\eta_{3, n}(z)
\end{array}\right)
$$

Since $V_{F}(z)$ is a symmetric matrix and $\mathcal{H}$ is Hermitian, thus the eigenvalues $\omega_{n}^{2}$ of $\mathcal{H}$ are real.

The Schrödinger-like equation (30) and the Hessian matrix $V_{F}(z)$ in Eq. (31) are obtained by taking a Taylor expansion of the potential $V\left(\phi_{j}\right)$ in terms of $\eta_{j}(t, z)$ and retaining the first order terms in the equations of motion.

\section{POTENTIAL MODEL WITH THREE SCALAR FIELDS}

As an application of this formalism, let us consider the following potential

$$
\begin{aligned}
V & =V\left(\phi_{1}, \phi_{2}, \phi_{3}\right)=\frac{1}{2}\left(\lambda \phi_{1}^{2}+\alpha \phi_{2}^{2}+\alpha \phi_{3}^{2}-\frac{m^{2}}{\lambda}\right)^{2} \\
& +\frac{1}{2}\left(-\alpha \phi_{1} \phi_{2}+\beta_{2} \phi_{3}^{2}-\beta_{2}\right)^{2} \\
& +\frac{1}{2} \phi_{3}^{2}\left(-\alpha \phi_{1}+2 \beta_{2} \phi_{2}+\alpha \beta_{1}\right)^{2}
\end{aligned}
$$

where $\alpha>0$ and $\beta_{i} \geq 0$. This is a generalized potential for three scalar fields which was constructed from the potential discussed recently [18], for two scalar fields. Note that the symmetry $Z_{2} \mathrm{x} Z_{2}$ is preserved only if $\phi_{2}=0$ or if $\beta_{1}=\beta_{2}=0$. 
The corresponding superpotential in a field theory model is given by

$$
W\left(\phi_{j}\right)=\frac{m^{2}}{\lambda} \phi_{1}-\frac{\lambda}{3} \phi_{1}^{3}-\alpha \phi_{1} \phi_{2}^{2}-\frac{\alpha}{2} \phi_{1} \phi_{3}^{2}+\beta_{2} \phi_{2} \phi_{3}^{2}-\beta_{2} \phi_{2}+\frac{1}{2} \alpha \beta_{1} \phi_{3}^{2} .
$$

It is required that $\phi_{j}$, satisfy the BPS state conditions, which are expressed by the following equations

$$
\begin{aligned}
\phi_{1}^{\prime} & =-\lambda \phi_{1}^{2}-\alpha \phi_{2}^{2}+\frac{m^{2}}{\lambda}-\alpha \phi_{3}^{2} \\
\phi_{2}^{\prime} & =-2 \alpha \phi_{1} \phi_{2}+\beta_{2} \phi_{3}^{2}-\beta_{2} \\
\phi_{3}^{\prime} & =\phi_{3}\left(-\alpha \phi_{1}+2 \beta_{2} \phi_{2}+\alpha \beta_{1}\right)
\end{aligned}
$$

and the superpotential $W\left(\phi_{j}\right)$ satisfy $\frac{\partial W}{\partial \phi_{j}}=U_{j}(j=1,2,3)$.

Note that the BPS states saturate the lower bound, so that $E_{B P S}=\left|W_{i j}\right|$ is the central charge of the realization of $N=1$ SUSY in $(1+1)$-dimensions. Thus, the vacua are determined by the extrema of the superpotential. Therefore, the condition

$$
\frac{\partial W}{\partial \phi_{j}}=0, \quad j=1,2,3
$$

provides the vacuum states $M_{i}=\left(\phi_{1 \mathrm{v}}, \phi_{2 \mathrm{~V}}, \phi_{3 \mathrm{~V}}\right)$ whose values must satisfy the following equations

$$
\begin{aligned}
& -\lambda \phi_{1}^{2}-\alpha \phi_{2}^{2}+\frac{m^{2}}{\lambda}-\frac{1}{2} \alpha \phi_{3}^{2}=0 \\
& -2 \alpha \phi_{1} \phi_{2}+\beta_{2} \phi_{3}^{2}-\beta_{2}=0 \\
& -\alpha \phi_{1}+2 \beta_{2} \phi_{2}+\alpha \beta_{1}=0 .
\end{aligned}
$$

In order to obtain an explicit form of the vacuum states, let us consider the cases $\phi_{2 \mathrm{~V}}=$ $\phi_{3 \mathrm{v}}=0$ and $\phi_{1 \mathrm{v}}=\phi_{3 \mathrm{v}}=0$, respectively. Thus, we obtain the four vacuum states, which are given by

$$
\begin{aligned}
& M_{1}=\left(-\frac{m}{\lambda}, 0,0\right) \\
& M_{2}=\left(\frac{m}{\lambda}, 0,0\right)
\end{aligned}
$$




$$
\begin{aligned}
& M_{3}=\left(0,-m \sqrt{\frac{1}{\lambda \alpha}}, 0\right) \\
& M_{4}=\left(0, m \sqrt{\frac{1}{\lambda \alpha}}, 0\right) .
\end{aligned}
$$

It is easy to verify that these vacuum states are satisfied by the equations given in (37), for $\beta_{2}=0$ and $\alpha \lambda>0$. Now, let consider $\phi_{1 \mathrm{v}}=\beta_{1}$ and $\phi_{2 \mathrm{v}}=0$ in Eq.(37). In this case, we obtain two additional vacuum states, which are

$$
\begin{aligned}
& M_{5}=\left(\beta_{1}, 0, \sqrt{\frac{2}{\alpha}\left(\frac{m^{2}}{\lambda}-\lambda \beta_{1}^{2}\right)}\right) \\
& M_{6}=\left(\beta_{1}, 0,-\sqrt{\frac{2}{\alpha}\left(\frac{m^{2}}{\lambda}-\lambda \beta_{1}^{2}\right)}\right),
\end{aligned}
$$

for $\beta_{2}=0,-\frac{m}{\lambda}<\beta_{1}<\frac{m}{\lambda}$ and $\alpha \neq 0$. Therefore, in this case the components of the tension are

$$
\begin{aligned}
& T_{12}=T_{21}=\frac{4}{3} \frac{m^{3}}{\lambda^{2}} \\
& T_{13}=T_{31}=T_{24}=T_{42}=T_{41}=T_{14}=T_{23}=T_{32}=\frac{2}{3} \frac{m^{3}}{\lambda^{2}} \\
& T_{15}=T_{51}=T_{25}=T_{52}=T_{16}=T_{61}=T_{26}=T_{62}=\left|\frac{2}{3} \frac{m^{3}}{\lambda^{2}}-\beta_{1}\left(\frac{m^{2}}{\lambda}-\frac{\lambda}{2} \beta_{1}^{2}\right)\right| \\
& T_{34}=T_{43}=0=T_{56}=T_{65} \\
& T_{35}=T_{53}=T_{45}=T_{54}=T_{36}=T_{63}=\left|\frac{\lambda}{3} \beta_{1}^{3}-\frac{m^{2}}{\lambda} \beta_{1}\right| .
\end{aligned}
$$

From the results given by Eq.(40), we see that the potential presents two non-topological sectors, which are non-BPS sectors, namely, $T_{34}$ and $T_{56}$, and twelve BPS topological sectors.

Now, let us specialize to the (3x3)-matrix superpotential, $\mathbf{W}$, with $\beta_{2}=0$, which is given by

$$
\mathbf{W}=\left(\begin{array}{ccc}
2 \lambda \phi_{1} & \alpha \phi_{2} & \alpha \phi_{3} \\
\alpha \phi_{2} & \alpha \phi_{1} & 0 \\
\alpha \phi_{3} & 0 & \alpha \beta_{1}-\alpha \phi_{1}
\end{array}\right)_{\mid \phi=\phi(z), \chi=\chi(z)}
$$

This superpotential satisfies the Ricatti equation associated with the non-diagonal fluctuation Hessian matrix, $V_{F}(z)$, which is written as 


$$
\mathbf{W}^{2}+\mathbf{W}^{\prime}=V_{F}(z)=\left(\begin{array}{ccc}
V_{F 11}(z) & V_{F 12}(z) & V_{F 13}(z) \\
V_{F 12}(z) & V_{F 22}(z) & V_{F 23}(z) \\
V_{F 13}(z) & V_{F 23}(z) & V_{F 33}(z)
\end{array}\right)_{\mid \phi=\phi(z), \chi=\chi(z)}
$$

where the elements of $V_{F}(z)$, denoted by $V_{F i j}(z)$, are given by the following relations

$$
\begin{aligned}
V_{F 11} & =6 \lambda^{2} \phi_{1}^{2}+\alpha^{2}\left(4 \phi_{2}^{2}+\phi_{3}^{2}\right)+2 \lambda\left(\alpha \phi_{2}^{2}+\frac{1}{2} \alpha \phi_{3}^{2}-\frac{m^{2}}{\lambda}\right) \\
V_{F 22} & =6 \alpha^{2} \phi_{2}^{2}+2 \alpha(2 \alpha+\lambda) \phi_{1}^{2}+\left(\alpha^{2}+4 \beta_{2}^{2}\right) \phi_{3}^{2}-2 \alpha \frac{m^{2}}{\lambda} \\
V_{F 12} & =V_{21}=4 \lambda \alpha \phi_{1} \phi_{2}+8 \alpha^{2} \phi_{1} \phi_{2}-2 \alpha\left(2 \beta_{2} \phi_{3}^{2}-\beta_{2}\right) \\
V_{F 13} & =V_{31}=2 \alpha(\lambda+\alpha) \phi_{1} \phi_{3}-4 \alpha \beta_{2} \phi_{2} \phi_{3}-2 \alpha^{2} \beta_{1} \phi_{3} \\
V_{F 33} & =\frac{3}{2} \alpha^{2} \phi_{3}^{2}+6 \beta_{2}^{2} \phi_{3}^{2}-2 \beta_{2}\left(2 \alpha \phi_{1} \phi_{2}+\beta_{2}\right)+\left(-\alpha \phi_{1}+2 \beta_{2} \phi_{2}+\alpha \beta_{1}\right)^{2} \\
V_{F 32} & =V_{F 23}=2 \alpha^{2} \phi_{2} \phi_{3}+4 \phi_{3} \beta_{2}\left(-\alpha \phi_{1}+2 \beta_{2} \phi_{2}^{2}+\alpha \beta_{1}\right) .
\end{aligned}
$$

The Ricatti equation (42) only holds for the BPS states. According to Witten's model[1, 4], we have

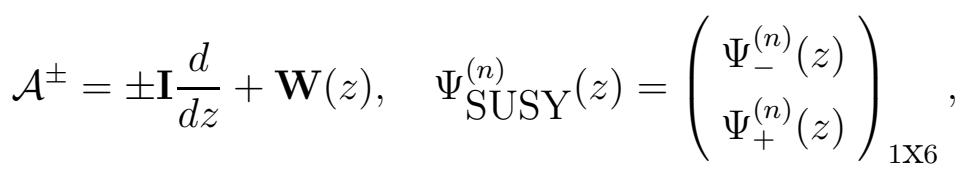

where $\Psi_{ \pm}^{(n)}(z)$ are three-component eigenfunctions. In this case, the graded Lie algebra of the SUSY QM for the BPS states may be realized as

$$
\begin{gathered}
H_{S U S Y}=\left[Q_{-}, Q_{+}\right]_{+}=\left(\begin{array}{cc}
\mathcal{A}^{+} \mathcal{A}^{-} & 0 \\
0 & \mathcal{A}^{-} \mathcal{A}^{+}
\end{array}\right)_{6 \mathrm{X} 6}=\left(\begin{array}{cc}
\mathcal{H}_{-} & 0 \\
0 & \mathcal{H}_{+}
\end{array}\right) \\
{\left[H_{S U S Y}, Q_{ \pm}\right]_{-}=0=\left(Q_{-}\right)^{2}=\left(Q_{+}\right)^{2}}
\end{gathered}
$$

where $Q_{ \pm}$are the (6x6) supercharges of the Witten model and is given by

$$
Q_{-}=\sigma_{-} \otimes \mathcal{A}^{-}, \quad Q_{+}=Q_{-}^{\dagger}=\left(\begin{array}{cc}
0 & \mathcal{A}^{+} \\
0 & 0
\end{array}\right)=\sigma_{+} \otimes \mathcal{A}^{+},
$$

with the intertwining operators, $\mathcal{A}^{ \pm}$, given in terms of (3x3)-matrix superpotential, Eq.(44), and $\sigma_{ \pm}=\frac{1}{2}\left(\sigma_{1} \pm i \sigma_{2}\right)$, with $\sigma_{1}$ and $\sigma_{2}$ being Pauli matrices. Note that the bosonic sector of 
$H_{S U S Y}$ is exactly the fluctuating operator given by $\mathcal{H}_{-}=\mathcal{H}=-\mathbf{I} \frac{d^{2}}{d z^{2}}+\mathbf{V}_{F}(z)$, where $\mathbf{V}_{-}=$ $\mathbf{V}_{F}(z)$ is the non-diagonal fluctuation Hessian matrix. The supersymmetric fluctuation partner operator of $\mathcal{H}_{-}$is

$$
\mathcal{H}_{+}=\mathcal{A}^{-} \mathcal{A}^{+}=\mathcal{A}^{+} \mathcal{A}^{-}+\left[\mathcal{A}^{-}, \mathcal{A}^{+}\right]=\mathcal{H}_{-}-\mathbf{W}^{\prime}(z),
$$

so that the SUSY partner is given by $\mathbf{V}_{+}=\mathbf{V}_{-}-\mathbf{W}^{\prime}(z)$.

The Ricatti equation given by (42) is reduced to a set of first-order coupled differential equations. In this case, the superpotential is not necessarily defined as $W(z)=\frac{1}{\psi_{-}^{(0)}} \frac{d}{d z} \psi_{-}^{(0)}(z)$, as in the case of a system described by a one-component wave function in the framework of SUSY QM[1, 4].

Therefore, as the zero-mode is associated with a three-component eigenfunction, $\Psi_{-}^{(0)}(z)$, one may write the matrix superpotential in the form [5]

$$
\frac{d}{d z} \Psi_{-}^{(0)}(z)=\mathbf{W} \Psi_{-}^{(0)}(z),
$$

from which we find the following zero mode eigenfunction

$$
\Psi_{-}^{(0)}=\left(\begin{array}{c}
U_{1}\left(\phi_{i}\right) \\
U_{2}\left(\phi_{i}\right) \\
U_{3}\left(\phi_{i}\right)
\end{array}\right)
$$

where $U_{i} \quad(i=1,2,3)$ are given by the BPS states (35).

Now, let us show that the $\omega_{n}^{2}$, s are non-negative. To do this, consider the bilinear form of $\mathcal{H}$ given by

$$
\mathcal{H}=\mathcal{A}^{+} \mathcal{A}^{-}
$$

where

$$
\mathcal{A}^{-}=\left(\mathcal{A}^{+}\right)^{\dagger}=\left(\begin{array}{ccc}
a_{1}^{-} & \mathcal{A}_{12}^{-} & \mathcal{A}_{13}^{-} \\
\mathcal{A}_{21}^{-} & a_{2}^{-} & \mathcal{A}_{23}^{-} \\
\mathcal{A}_{31}^{-} & \mathcal{A}_{32}^{-} & a_{3}^{-}
\end{array}\right)=\left(\begin{array}{ccc}
a_{1}^{-} & 0 & 0 \\
0 & a_{2}^{-} & 0 \\
0 & 0 & a_{3}^{-}
\end{array}\right)+\mathcal{R}\left(\phi_{i}\right),
$$

with the obvious identification of the elements of $\mathcal{R}\left(\phi_{i}\right)$ and the following expressions for the operators that appear in the analysis of classical stability associated with a single field [2] 


$$
\begin{aligned}
& a_{1}^{-}=-\frac{d}{d z}+2 \lambda \phi_{1}, \\
& a_{2}^{-}=-\frac{d}{d z}+\alpha \phi_{1}, \\
& a_{3}^{-}=-\frac{d}{d z}+\alpha \phi_{1}-\alpha \beta_{1},
\end{aligned}
$$

where

$$
\mathcal{A}_{12}^{-}=\alpha \phi_{2}=\mathcal{A}_{21}^{-}, \quad \mathcal{A}_{13}^{-}=\alpha \phi_{3}=\mathcal{A}_{31}^{-}, \quad \mathcal{A}_{23}^{-}=0=\mathcal{A}_{32}^{-} .
$$

Since $a_{j}^{+}=\left(a_{j}^{-}\right)^{\dagger}$ and hence $\mathcal{A}^{+}=\left(\mathcal{A}^{-}\right)^{\dagger}$, we find

$$
\left(\mathcal{A}^{+} \mathcal{A}^{-}\right)_{j j}=-\frac{d^{2}}{d z^{2}}+\frac{\partial^{2}}{\partial \phi_{j}^{2}} V,
$$

which are exactly the diagonal elements of $\mathcal{H}$. It is worth calling attention to the fact that the linear stability is satisfied, which means that

$$
\omega_{n}^{2}=<\mathcal{H}>=<\mathcal{A}^{+} \mathcal{A}^{-}>=\left(\mathcal{A}^{-} \tilde{\Psi}_{n}\right)^{\dagger}\left(\mathcal{A}^{-} \tilde{\Psi}_{n}\right) \geq 0
$$

and therefore the $\omega_{n}^{2}$, s are non-negative.

\section{PROJECTIONS ON SCALAR FIELDS}

Let us now consider a projection on the $\left(\phi_{1}, \phi_{2}\right)$ plane in order to find an explicit form

of domain walls using the trial orbit method. In this case, if we choose $\phi_{3}=0$ in Eq. (35) and the following trial orbit

$$
G\left(\phi_{1}, \phi_{2}\right)=c_{1} \phi_{1}^{2}+c_{2} \phi_{2}^{2}+c_{3}=0
$$

we get from $\frac{d G}{d z}=\frac{\partial G}{\partial \phi_{1}} \phi_{1}^{\prime}+\frac{\partial G}{\partial \phi_{2}} \phi_{2}^{\prime}=0$ and using the BPS states (35), that $c_{1}=1, c_{2}=\frac{\alpha}{2(\lambda-\alpha)}$ and $c_{3}=-\frac{m^{2}}{\lambda^{2}}$. Thus, the resulting elliptical orbit is

$$
\phi_{1}^{2}+\frac{\alpha}{(\lambda-2 \alpha)} \phi_{2}^{2}=\frac{m^{2}}{\lambda^{2}}
$$

or

$$
\frac{\lambda^{2}}{m^{2}} \phi_{1}^{2}+\frac{\lambda^{2}}{2 m^{2}} \phi_{2}^{2}=1,
$$


for $\alpha=\frac{\lambda}{4}$. These provide the following BPS solutions

$$
\begin{aligned}
\phi_{1}(z) & =\frac{m}{\lambda} \tanh \left(\frac{m}{2} z\right) \\
\phi_{2}(z) & = \pm \sqrt{2} \frac{m}{\lambda} \operatorname{sech}\left(\frac{m}{2} z\right) \\
\phi_{3}=0 &
\end{aligned}
$$

which connect the vacua $\left(\frac{m}{\lambda}, 0,0\right)$ and $\left(-\frac{m}{\lambda}, 0,0\right)$. Note that

$$
\begin{gathered}
\phi_{1} \rightarrow \pm \frac{m}{\lambda}, \text { for } \quad z \rightarrow \pm \infty \\
\phi_{2} \rightarrow 0, \text { for } z \rightarrow \pm \infty
\end{gathered}
$$

This result corresponds to the same one obtained recently[18] for BPS solutions when two scalar fields are taken into account.

In this situation, the matrix superpotential in $\operatorname{SUSY}$ QM, W(z), becomes

$$
\mathbf{W}(z)=-\frac{m}{2}\left(\begin{array}{ccc}
4 \tanh \left(\frac{m}{2} z\right) & \pm \sqrt{2} \operatorname{sech}\left(\frac{m}{2} z\right) & 0 \\
\pm \sqrt{2} \operatorname{sech}\left(\frac{m}{2} z\right) & \tanh \left(\frac{m}{2} z\right) & 0 \\
0 & 0 & 4
\end{array}\right)
$$

where we have used the BPS states in terms of a projection on the $(\phi, \chi)$ plane. This provides the following bosonic zero-mode

$$
\frac{d}{d z} \Psi_{-}^{(0)}(z)=\mathbf{W} \Psi_{-}^{(0)}(z) \Rightarrow \Psi_{-}^{(0)}(z)=N\left(\begin{array}{c}
\operatorname{sech}^{2}\left(\frac{m}{2} z\right) \\
\pm \sqrt{2} \tanh \left(\frac{m}{2} z\right) \operatorname{sech}\left(\frac{m}{2} z\right) \\
0
\end{array}\right)
$$

for $\alpha=\frac{\lambda}{4}$, where $N$ is the normalization constant. However, in Ref. [18] the relation between $\lambda$ and $\alpha$ is given by $\alpha=\frac{\lambda}{2}$.

\section{CONCLUSIONS}

In this paper, we considered the classical stability analysis for BPS domain walls associated with a potential model of three coupled real scalar fields, which obeys the non-ordinary 
supersymmetry (SUSY). The approach of effective quantum mechanics provides a realization of SUSY algebra in the three-domain wall sector of the non-relativistic formalism.

The components of the tension given in (40) were deduced from the charge central properties in the model that present $N=1$ SUSY. From a three-field specific potential model we found two null tensions which correspond to non-topological sectors, and other topological sectors, which depend on the manifold of vacuum states, $T_{i j}=\left|W\left[M_{j}\right]-W\left[M_{i}\right]\right|$, where $M_{i}$ and $M_{j}$ represent the vacuum states.

We have shown that the positive potentials with a square form lead to three-component non-negative normal modes $\omega_{n}{ }^{2} \geq 0$, analogous to the case with a single field [2], so that the linear stability of the Schrödinger-like equations is ensured.

We have seen that domain walls associated with the three-field potentials have features that are not present in the one-field models. The BPS states which connect the vacua exist within the stability domain and minimize the energy. Thus, they provide a realization of the supersymmetric quantum mechanical algebra for three-component eigenfunctions. From the stability equation, we have found an expression for the matrix superpotential, satisfying the Ricatti equation, within the context of unidimensional quantum mechanics.

We also deduced an (3x3)-matrix explicit form of the SUSY QM superpotential from a field-theoretic superpotential model in (1+1)-dimensions. A general three-component zeromode eigenfunction is deduced, but its explicit form is found only for the projection on the $\left(\phi_{1}, \phi_{2}\right)$ plane, $\phi_{3}=0$, and for $\alpha=\frac{\lambda}{4}$, under which the original superpotential becomes harmonic.

Recently, in the context of a three-field potential model was considered an hexagonal network of static classical configuration inside a topological soliton. Also, the 1/4 BPS equations of domain wall junction were first obtained by Gibbons and Townsend [21] and independently by Carrol et al. [22]. We point out that the superpotential model investigated here can be applied to implement new string junctions by extended BPS domain walls[23].

\section{Acknowledgments}

RLR would like to acknowledge S. Alves for hospitality at CCP-CBPF of Rio de JaneiroRJ, Brazil, where the part of this work was carried out and to J. A. Helaÿel-Neto and I. V. Vancea for many stimulating discussions. This work was partially supported by Conselho Nacional de Desenvolvimento Científico e Tecnológico $(\mathrm{CNPq})$, by Fundação de Apoio à Pesquisa do Estado da Paraíba (FAPESQ)/PRONEX/CNPq and by Fundação de Apoio à 
Ciência e Tecnologia do Espírito Santo(FAPES)/PRONEX/CNPq.

[1] E. Witten, Nucl. Phys. B185, 513 (1981).

[2] V. Gomes Lima, V. Silva Santos and R. de Lima Rodrigues, Phys. Lett. A298, 91 (2002).

[3] T. Vachaspati, Phys. Rev. D69, 043510 (2004).

[4] L. E. Gendenshtein, JETP Lett. 38, 356 (1983); L. E. Gendenshtein and I. V. Krive, Sov. Phys. Usp. 28, 645 (1985); A. Lahiri, P. K. Roy, and B. Bagchi, Int. J. Mod. Phys. A5, 1383 (1990); F. Cooper, A. Khare, and U. Sukhatme, Phys. Rep. 251, 267 (1995).

[5] A. Andrianov, F. Cannata, M. V. Ioffe and D. N. Nishnianidze J. Phys. A: Math. Gen. 30, 5037 (1997); T. Fukui, Phys. Lett. A178, 1 (1993).

[6] R. de Lima Rodrigues, The Quantum Mechanics SUSY Algebra: an Introductory Review, Monograph CBPF MO-03/01, hep-th/0205017 and references therein.

[7] R. de Lima Rodrigues, P. Barbosa da Silva Filho, and A. N. Vaidya, Phys. Rev. D58, 125023 (1998).

[8] H. Falomir and P. A. G. Pisani, J. Phys. A38, 4665 (2005).

[9] T. K. Das and B. Chakrabarti, J. Phys. A32, 2387 (1999); M. Robnik, J. Phys. A30, 1287 (1997).

[10] R. Jackiw, Rev. Mod. Phys. 49, 681 (1977).

[11] R. Rajaraman, Phys. Rev. Lett., 42, 200 (1979); R. Rajaraman, Solitons and Instantons, (North-Holland, Amsterdam, 1982).

[12] S. Coleman, Aspects of Symmetry, (Cambridge University, London, 1985).

[13] A. P. Balachandran, G. Marmo, B. S. Skagerstam and A. Stern, Classical Topology and Quantum States, (World Scientific, Singapore, 1991).

[14] E. J. Weinberg, Annual Rev. Nucl. Part. Sci. 42, 177 (1992).

[15] E. B. Bogomol'nyi, Sov. J. Nucl. Phys. 24, 449 (1976).

[16] M. K. Prasad, C. H. Sommerfield, Phys. Rev. Lett. 35, 760 (1975).

[17] A. Alonso Izquierdo, M. A. González León and J. Mateos Guilarte, Phys. Rev. D65, 085012 (2002).

[18] G. de Sousa Dias, E. L. Graça and R. de Lima Rodrigues, Int. J. Mod. Phys. A22, 731 (2007).

[19] A. Ritz, M. Shifman, A. Vainshtein, and M. Voloshin, Phys. Rev., D63, 065018 (2001). 
[20] K. Shizuya, Phys. Rev. D69, 065021 (2004).

[21] G. W. Gibbons, P. K. Townsend, Phys. Rev. Lett. 83, 1727 (1999), hep-th/9905196.

[22] S. M. Carroll, S. Hellerman and M. Trodden, Phys. Rev. 61D, 065001 (2000).

[23] R. A. Battye and P. M. Sutcliffe, Phys. Rev. Lett. 86, 3989 (2001). 